\title{
REVIEW
}

\section{The acute respiratory management of cervical spinal cord injury in the first 6 weeks after injury: a systematic review}

\author{
S Berney ${ }^{1}$, P Bragge $^{2}$, C Granger ${ }^{1}$, H Opdam $^{3}$ and L Denehy ${ }^{4}$ \\ ${ }^{1}$ Physiotherapy Department, Austin Hospital, Melbourne, Victoria, Australia; ${ }^{2}$ National Trauma Research Institute, \\ Monash University, Melbourne, Victoria, Australia; ${ }^{3}$ Department of Intensive Care, Austin Hospital, Melbourne, Victoria, \\ Australia and ${ }^{4}$ School of Health Sciences, The University of Melbourne, Parkville, Victoria, Australia
}

\begin{abstract}
Study design: Systematic review.
Objectives: Identify, evaluate, and synthesize evidence regarding the effectiveness of various treatment strategies for the respiratory management of acute tetraplegia.

Setting: Melbourne, Australia.

Methods: A search of multiple electronic databases (Medline, Cinahl, EMBASE, Cochrane Library, Web of Science, http://www.guideline.gov and http://www.icord.org/scire) was undertaken accompanied by the reference lists of all relevant articles identified. Methodological quality was assessed using the Newcastle-Ottawa Scale and the PEDro Scale. Descriptive analysis was performed.

Results: Twenty-one studies including 1263 patients were identified. The majority of the studies were case series $(n=13)$. A variety of interventions were used for the management of respiratory complications. Mortality ( $A R R=0.4,95 \%$ confidence interval $(\mathrm{Cl}) 0.18,0.61)$, the incidence of respiratory complications $(\mathrm{ARR}=0.36,95 \% \mathrm{Cl}(0.08,0.58)$ ), and requirement for a tracheostomy $(A R R=0.18,95 \% \mathrm{Cl}(-0.05,0.4))$ were significantly reduced by using a respiratory protocol. A clinical pathway reduced duration of mechanical ventilation by 6 days $95 \% \mathrm{Cl}(-0.56,12.56)$, intensive care unit length of stay by 6.8 days $95 \% \mathrm{Cl}(0.17-13.77)$ and costs. Intubation, mechanical ventilation, and tracheostomy are the mainstay of respiratory management for complete injuries above the level of C5. Conclusion: This review showed a clinical pathway with a structured respiratory protocol that includes a combination of treatment techniques provided regularly is effective in reducing respiratory complications and cost. The overall study quality was moderate and further studies using specific interventions that target respiratory complications are associated with specific regions of the cervical spine using more methodologically rigorous designs are required.
\end{abstract}

Spinal Cord (2011) 49, 17-29; doi:10.1038/sc.2010.39; published online 20 April 2010

Keywords: respiratory management; pulmonary complications; tetraplegia; intensive care

\section{Introduction}

Spinal cord injury (SCI) resulting in tetraplegia has a profound effect on respiratory function. ${ }^{1}$ Pulmonary complications are the leading cause of morbidity and death both in the shortand longer-term after injury. ${ }^{2-4}$ In the acute hospitalization phase respiratory complications are highly prevalent with $84 \%$ of patients with $\mathrm{C} 1-4$ and $60 \%$ of those with $\mathrm{C} 5-\mathrm{C} 8$ injuries experiencing respiratory compromise. ${ }^{3}$ The number of respiratory complications during this acute phase contributes significantly to both hospital length of stay and costs. ${ }^{4}$

In addition to muscle paralysis, a period of spinal shock occurs immediately after a traumatic cervical SCI, resulting in flaccid paralysis of muscles below the level of the cord

Correspondence: S Berney, C/O Physiotherapy Level 3 HSB, Austin Hospital, 145 Studley Road, PO Box 5555 Heidelberg, Melbourne, Victoria 3084, Australia. E-mail: sue.berney@austin.org.au

Received 3 February 2010; accepted 12 March 2010; published online 20 April 2010 injury ${ }^{5,6}$ that can last from a period of 4 weeks $^{7}$ up to several months. ${ }^{6}$ The severity of spinal shock is related to the severity of the injury and is correlated with the incidence of respiratory complications. ${ }^{6,8}$ The impact of spinal shock on respiratory function can be so severe as to require a transient need for an artificial airway and mechanical ventilatory assistance. $^{2}$ As spinal shock resolves the flaccid paralysis of muscles is replaced by spasticity and the chest wall becomes rigid resulting in an improvement in respiratory function particularly during inspiration. ${ }^{5,9}$ As a result respiratory complications in the acute phase follow a predictable time course developing within the first 5 days and lasting up to 5 weeks post injury as spinal shock resolves. ${ }^{3}$ Aggressive respiratory management has been advocated for the prevention and treatment of pulmonary complications and has been associated with improved outcomes. ${ }^{2,10,11}$

The frequency of respiratory complications is correlated with injury level and severity, associated injuries, and the age 
and pre-existing co-morbidities of the patient. ${ }^{8}$ The nature of the pulmonary complication can also vary with the level of the injury; with high injuries (C1-C4) being more likely to develop pneumonia and lower cervical injuries atelectasis. ${ }^{3}$ Respiratory management encompasses a variety of strategies including airway management, weaning protocols, respiratory care protocols, and physiotherapy intervention. ${ }^{6,12}$ Guidelines for respiratory management after SCI were published in $2005,{ }^{13}$ however, these recommendations were not specific to the acute care setting and relied on evidence that did not pertain to the acute SCI population. The aims of this systematic review are to identify, evaluate, and synthesize evidence regarding the effectiveness of various treatment strategies for the respiratory management of acute tetraplegia.

\section{Materials and methods}

Data sources and searches

Electronic literature searching was conducted in Medline (1950-2008), Cinahl (1982-2008), EMBASE (1980-2008), the Cochrane Library (2008), Web of Science (1900-1914-2008), http://www.guideline.gov and http://www.icord.org/scire/ chapters.php on 20 October 2008 using the following terms: respiration disorders, mechanical ventilators/artificial respiration, respiratory insufficiency, tetraplegia/quadriplegia, spinal cord injuries, intubation/intratracheal, tracheotomy, tracheostomy, respiratory dysfunction, and respiratory management. Terms were mapped to the appropriate Medical Subject Heading (MeSH) and to the EMTREE subject headings in EMBASE and 'exploded'. The search was limited to articles published in English. In addition to this electronic search the reference list of retrieved articles as well as personal files that included invited reviews and clinical guidelines were hand searched to identify further relevant citations.

\section{Study selection}

Inclusion and exclusion criteria are detailed in Table 1 . We included study designs without a comparison group because of the paucity of comparative studies on this topic. Case studies and conference abstracts were excluded as they provided insufficient information to evaluate methodological quality.

Titles retrieved from searching and their reference lists were screened against the inclusion and exclusion criteria to identify potentially relevant papers; these papers were then reviewed in abstract form and potentially relevant abstracts were selected for full text analysis, from which the final selection was made. Each of the steps was performed independently by two authors (SB and LD) and full text review by two authors ( $\mathrm{SB}$ and $\mathrm{CG}$ ). If any discrepancy occurred during the review of the selected articles a third author (PB) adjudicated. Agreement between the reviewers was estimated using the Kappa statistic.

\section{Study design and quality}

Studies were evaluated using two methods. Study design was classified according to the National Health and Medical Research Council (NHMRC) Hierarchy of Evidence ${ }^{14}$ (Table 1). Using this method, study design is rated from I-IV with I being the strongest providing evidence from a systematic review of randomized controlled trials (RCTs) and IV being the weakest design providing evidence from a case series. Using a standardized data collection form, the methodological quality of RCTs was scored using the PEDro Scale. This scale is a validated quality assessment tool for RCTs. ${ }^{15}$ The scale is scored out of 10 and specifically examines the internal validity and adequacy of statistical information presented. The methodological quality of relevant non-randomized and observational studies was assessed using the Newcastle-Ottawa Scale (NOS). ${ }^{16}$ The NOS is a validated instrument specifically designed to assess the quality of observational studies in a systematic review and has been previously used in intensive care unit (ICU) populations. ${ }^{17}$ The NOS evaluates three domains of methodology that include nine scored criteria: the selection of study groups (score range 0-4), the comparability of groups (score range 0-2), and the degree of certainty of the outcomes (score range 0-3) (Table 2).

Table 1 Inclusion and exclusion criteria

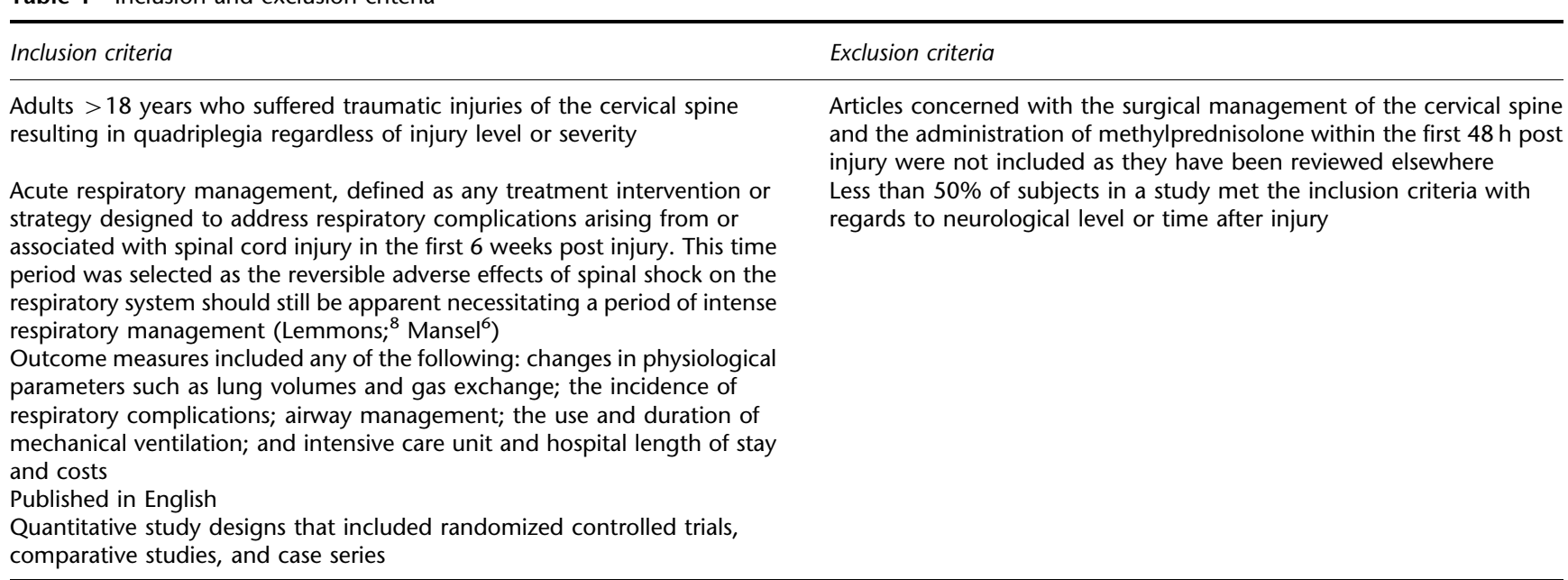


Table 2 Scoring criteria used for synthesizing results of studies in the review

\begin{tabular}{lcc}
\hline & $\begin{array}{c}\text { Newcastle- } \\
\text { Ottawa Scale }\end{array}$ & $\begin{array}{c}\text { PEDro } \\
\text { Scale }\end{array}$ \\
\hline $\begin{array}{l}\text { Strong evidence - consistent findings among } \\
\text { multiple high-quality studies }\end{array}$ & $6 / 9$ & $6-10 / 10$ \\
$\begin{array}{l}\text { Moderate evidence - consistent findings among } \\
\text { multiple lower quality studies and/or one } \\
\text { high-quality study }\end{array}$ & $4-5 / 9$ & $4-5 / 10$ \\
$\begin{array}{l}\text { Limited evidence-one lower quality study } \\
\begin{array}{l}\text { Conflicting evidence-inconsistent findings } \\
\text { among multiple studies }\end{array}\end{array}$ & $<4$ & $<4$ \\
\begin{tabular}{l} 
No evidence-no evidence among studies \\
\hline
\end{tabular} & & \\
\hline
\end{tabular}

Newcastle-Ottawa Scale used for non-randomized controlled trials and PEDro Scale used for randomized controlled trial.

Table 3 Information and outcome measures extracted from studies

\begin{tabular}{ll}
\hline Patient information & Outcome measures \\
\hline Patient demographic characteristics & $\begin{array}{l}\text { Change in physiological variables } \\
\text { such as gas exchange or lung } \\
\text { volumes } \\
\text { Intensive care unit and hospital } \\
\text { length of stay }\end{array}$ \\
Injury level & $\begin{array}{l}\text { The incidence of respiratory } \\
\text { complications }\end{array}$ \\
Injury severity & Chest radiograph changes \\
Type of respiratory management & The use and duration of \\
Dose of respiratory management & mechanical \\
(when available) & ventilation \\
& Airway management \\
& Costs
\end{tabular}

\section{Data extraction and synthesis}

The following information was extracted from relevant studies when available (Table 3). Mean differences and 95\% confidence intervals (CIs) and odds ratios or relative risk and 95\% CIs were calculated if they were not provided. Owing to the heterogeneity of interventions and outcome measures, outcomes were not pooled.

Results of the included studies were narratively synthesized. An established protocol was used to aid interpretation of the results of the review for the $\operatorname{NOS}^{18}$ consistent with previous systematic reviews ${ }^{19}$ and for the PEDro Scale ${ }^{20}$ (Table 2).

\section{Results}

\section{Study selection}

The initial search retrieved 336 citations. The subsequent review of titles, abstracts, and full length articles yielded 21 reports. All findings refer, unless otherwise stated, to the remaining 21 papers (Figure 1).

Study design and quality

The 21 included papers comprised 1 RCT, 3 cohort studies, 3 case-control studies, and 14 retrospective case series reports. The methodological quality of the studies was moderate

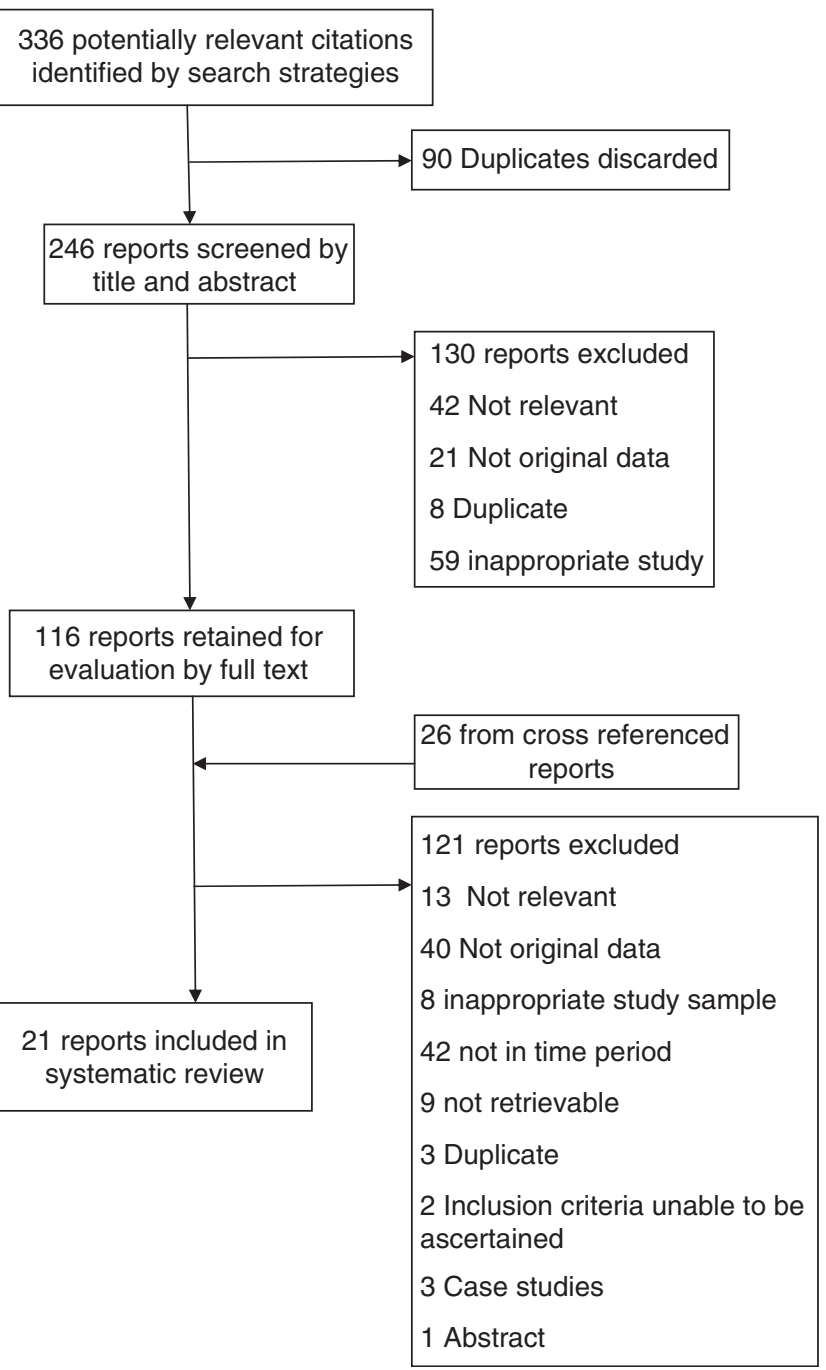

Figure 1 Flow of studies through the review.

overall with one RCT achieving a PEDro score of three and eight studies achieving an NOS $>$ six and with mean score of five (Table 4). In general, as most studies were retrospective case series there was no score attributable for comparability; however, population selection, outcome assessment, and follow-up were satisfactory (Table 5).

Data extraction and synthesis

The mean sample size of the included studies was 68.52 (range 3-186) and a total of 1415 patients were included across all studies. Subjects were predominantly male with a complete injury between C4 and C6. Although all studies were single centre they reflected an international experience with $52 \%$ of studies from North America, 33\% from Europe, and $14 \%$ from Australia. Specialist SCI centres were involved in $85 \%$ of the studies. Kappa statistics for inter-reviewer selection of potentially relevant titles and abstracts and full text articles were 0.87 and 0.77 , respectively, showing substantial agreement. 
Table 4 Study quality for randomized controlled trial

\begin{tabular}{ccccccccccccc}
\hline Study & $\begin{array}{c}\text { Random } \\
\text { allocation }\end{array}$ & $\begin{array}{c}\text { Concealed } \\
\text { allocation }\end{array}$ & $\begin{array}{c}\text { Groups } \\
\text { similar at } \\
\text { baseline }\end{array}$ & $\begin{array}{c}\text { Participant } \\
\text { blinding }\end{array}$ & $\begin{array}{c}\text { Therapist } \\
\text { blinding }\end{array}$ & $\begin{array}{c}\text { Assessor } \\
\text { blinding }\end{array}$ & $\begin{array}{c}<15 \% \\
\text { dropouts }\end{array}$ & $\begin{array}{c}\text { Intention- } \\
\text { to-treat } \\
\text { analysis }\end{array}$ & $\begin{array}{c}\text { Between-group } \\
\text { difference } \\
\text { reported }\end{array}$ & $\begin{array}{c}\text { Point estimate } \\
\text { and variability } \\
\text { reported }\end{array}$ & $\begin{array}{c}\text { Total } \\
\text { (0-10) }\end{array}$ & $\begin{array}{c}\text { NHMRC } \\
\text { grade of } \\
\text { evidence }\end{array}$ \\
\hline Pllastrini & Yes & No & Yes & No & No & No & No & No & No & Yes & 3 & II \\
\hline
\end{tabular}

Study quality and design for non randomized controlled trials

\begin{tabular}{|c|c|c|c|c|c|c|}
\hline $\begin{array}{l}\text { Newcastle-Ottawa } \\
\text { Scale }\end{array}$ & Selection & Comparability & Outcome & Total/9 & Design & $\begin{array}{l}\text { NHMRC grade } \\
\text { of evidence }\end{array}$ \\
\hline Gregoretti et al. ${ }^{22}$ & 4 & 2 & 3 & 9 & Case-control & III-2 \\
\hline Berney et al. ${ }^{39}$ & 4 & 2 & 3 & 9 & Case-control & III-2 \\
\hline Vitaz et al. ${ }^{33}$ & 4 & 1 & 3 & 8 & Retro cohort & III-3 \\
\hline Braun et al. ${ }^{21}$ & 2 & 2 & 2 & 6 & Case-control & III-2 \\
\hline Berney et al. ${ }^{35}$ & 3 & 0 & 3 & 6 & Case series & IV \\
\hline Harrop et al. ${ }^{36}$ & 3 & 0 & 3 & 6 & Case series & IV \\
\hline Hassid et al..$^{30}$ & 3 & 0 & 3 & 6 & Case series & IV \\
\hline Tromans et al. ${ }^{31}$ & 3 & 0 & 3 & 6 & Case series & IV \\
\hline Velmahos et al. ${ }^{32}$ & 3 & 0 & 3 & 6 & Case series & IV \\
\hline McMichan et al. ${ }^{10}$ & 3 & 0 & 2 & 5 & Retro cohort & III-3 \\
\hline Lerman and Weiss ${ }^{25}$ & 2 & 0 & 3 & 5 & Case series & IV \\
\hline Como et al. $^{37}$ & 2 & 0 & 3 & 5 & Case series & IV \\
\hline Romero et al. ${ }^{38}$ & 2 & 0 & 3 & 5 & Case Series & IV \\
\hline Bellamy et al. $^{34}$ & 2 & 0 & 2 & 4 & Cohort & $\mathrm{III}-2$ \\
\hline Hornstein and Ledsome ${ }^{24}$ & 2 & 0 & 2 & 4 & Case series & IV \\
\hline Stiller et al. ${ }^{27}$ & 2 & 0 & 2 & 4 & Case series & IV \\
\hline Green et al. ${ }^{28}$ & 2 & 0 & 2 & 4 & Case series & IV \\
\hline Gardner et al. ${ }^{40}$ & 2 & 0 & 1 & 3 & Case series & IV \\
\hline Gupta et $a l^{23}$ & 1 & 0 & 0 & 1 & Case series & IV \\
\hline Hachen et al. ${ }^{29}$ & 0 & 0 & 1 & 1 & Case series & IV \\
\hline
\end{tabular}

Abbreviation: NHMRC, National Health and Medical Research Council.

Respiratory management interventions and protocols

A variety of treatment techniques, management strategies, and protocols including a clinical pathway were used for the treatment and management of respiratory complications in the studies (Table 6). Protocols included a combination of modalities at varied intensity. The clinical pathway was restricted to a description of timing of processes of care.

\section{Results: physiological outcomes}

Seven studies evaluated physiological outcomes. ${ }^{21-27}$ There was moderate evidence to support the use of an assist cough to improve cough efficacy by significantly increasing peak expiratory flow ${ }^{21}$ and that the use of trans-tracheal open ventilation (mechanical ventilation through minitracheostomy) ${ }^{22}$ was effective in maintaining gas exchange and respiratory mechanics for an initial $24 \mathrm{~h}$ period. There was also moderate evidence for the provision of respiratory muscle resistance training to improve maximum inspiratory pressure ${ }^{24,25}$ and vital capacity. ${ }^{25}$ Two studies reported the effects of intermittent positive pressure breathing on lung volume. $^{25,27}$ The intervention was used differently in each study, one as part of a treatment package ${ }^{25}$ and the other as a sole treatment. ${ }^{27}$ The studies, both of low quality, reported a significant improvement in vital capacity. However, when intermittent positive pressure breathing was used as a sole intervention the improvement in lung volume was small and of limited clinical significance. There was limited evidence because of poor study quality to support the use of insufflation/exsufflation ${ }^{26}$ and minitracheostomy. ${ }^{23}$

\section{Incidence of respiratory complications}

Seven papers reported the effect of various interventions on the incidence of respiratory complications. ${ }^{10,28-33}$ Respiratory complications were defined as pneumonia, atelectasis, or respiratory insufficiency - a diagnosis that included respiratory failure, pneumonia, respiratory infection, and oxygen desaturation. Five of the studies were retrospective case series ${ }^{10,28-32}$ and the remaining two studies were cohort designs that used a retrospective control group. ${ }^{10,33}$ There was moderate evidence that a comprehensive clinical pathway $^{33}$ and a respiratory protocol ${ }^{10}$ reduced respiratory complications. Similar to Lerman and Weiss, McMichan et al. used a package of respiratory interventions that showed a significant reduction in the incidence of atelectasis compared with a retrospective control group $(\mathrm{ARR}=0.36$, $95 \%$ CI $(0.08,0.58))$ with a number needed to treat of three. In addition, there was a decreased requirement for tracheostomy $(\mathrm{ARR}=0.18,95 \% \mathrm{CI}(-0.05,0.4))$ with a number needed to treat of six. There was moderate evidence, albeit from a small case series, that showed non-invasive ventilation (NIV) was useful for the treatment of respiratory failure and may avoid the need for intubation. ${ }^{31}$ Given the quality of the study and design, ${ }^{28}$ there is little credible evidence to support the routine use of kinetic therapy (continuous bed 
Table 5 Study characteristics for randomized control trials/case control and cohort studies

\begin{tabular}{|c|c|c|c|c|c|c|c|c|}
\hline \multirow[t]{2}{*}{ Citation } & \multicolumn{2}{|c|}{ Subjects } & \multicolumn{2}{|c|}{ Injury level range/mode } & \multicolumn{2}{|c|}{$A S I A$} & \multirow{2}{*}{$\begin{array}{l}\text { Setting } \\
\text { Site } \\
\text { Specialist centre }\end{array}$} & \multirow[t]{2}{*}{ Outcome measure } \\
\hline & $\begin{array}{l}\text { Intervention } \\
N= \\
\mathrm{C} / \mathrm{I} \\
\text { Gender } \mathrm{M} / \mathrm{F} \\
\text { Mean age } \\
\text { (years) }\end{array}$ & $\begin{array}{l}\text { Control } \\
N= \\
C / I \\
\text { Gender } M / F \\
\text { Mean age } \\
\text { (years) }\end{array}$ & Intervention & Control & Intervention & Control & & \\
\hline Bellamy et $a .^{34}$ & $\begin{array}{l}54 \\
30 / 241 \\
44 / 10 \\
\text { Age NS }\end{array}$ & $\begin{array}{l}8 \\
\text { CSCI NS } \\
\text { Gender NS } \\
\text { Age NS }\end{array}$ & $\begin{array}{l}\text { C3-C7 Mode } \\
\text { NS }\end{array}$ & NS & $\begin{array}{l}\text { A } \\
\text { I: NS }\end{array}$ & No deficit & $\begin{array}{l}\mathrm{SCl} \\
\text { specialist } \\
\mathrm{ICU}\end{array}$ & Airway management \\
\hline Vitaz et al. ${ }^{33}$ & $\begin{array}{l}36 \\
\text { C: NS } \\
\text { Gender NS } \\
33 \pm 15\end{array}$ & $\begin{array}{l}22 \\
\text { C: NS } \\
\text { Gender NS } \\
34 \pm 10\end{array}$ & $\begin{array}{l}\text { C1-T5 } \\
\text { Mode C6/7 }\end{array}$ & $\begin{array}{l}\text { C1-T5 } \\
\text { Mode }=\mathrm{C} 5 / 6\end{array}$ & $\begin{array}{l}\text { AMS }= \\
22 \pm 22\end{array}$ & $\begin{array}{l}\text { AMS }= \\
19 \pm 24\end{array}$ & $\mathrm{ICU}$ & $\begin{array}{l}\text { Rate of pneumonia, ventilator } \\
\text { days, ICU and hospital length } \\
\text { of stay, cost comparison }\end{array}$ \\
\hline McMichan et al. ${ }^{10}$ & $\begin{array}{l}22 \\
\mathrm{C}: \mathrm{NS} \\
18 / 4 \\
24 \pm 3\end{array}$ & $\begin{array}{l}22 \\
C: \text { NS } \\
20 / 2 \\
29 \pm 3\end{array}$ & $\begin{array}{l}\text { C4-C8 } \\
\text { Mode NS }\end{array}$ & $\begin{array}{l}\text { C3-C8 } \\
\text { Mode NS }\end{array}$ & NS & NS & $\mathrm{ICU}$ & $\begin{array}{l}\text { Incidence of mortality, } \\
\text { atelectasis, need for } \\
\text { mechanical ventilation, } \\
\text { and tracheostomy }\end{array}$ \\
\hline Gregoretti et al. ${ }^{22}$ & $\begin{array}{l}10 \\
\text { C: NS } \\
10 / 0 \\
34\end{array}$ & $\begin{array}{l}10 \\
\mathrm{C}: \mathrm{NS} \\
10 / 0 \\
34\end{array}$ & $\begin{array}{l}\text { C4-6 } \\
\text { Mode }=C 5\end{array}$ & $\begin{array}{l}\text { C4-6 } \\
\text { Mode }=C 5\end{array}$ & A & A & $\mathrm{ICU}$ & $\begin{array}{l}\text { Gas exchange, respiratory } \\
\text { mechanics, respiratory rate }\end{array}$ \\
\hline Braun et al. ${ }^{21}$ & $\begin{array}{l}13 \\
C: 13 \\
11 / 2 \\
30.9 \pm 17.3\end{array}$ & $\begin{array}{l}13 \\
C: 13 \\
11 / 2 \\
30.9 \pm 17.3\end{array}$ & $\begin{array}{l}\text { C4-T6 } \\
\text { Mode level } \\
\text { NS }\end{array}$ & $\begin{array}{l}\text { C4-T6 } \\
\text { Mode level } \\
\text { NS }\end{array}$ & A & A & $\begin{array}{l}\text { Acute } \\
\text { ward }\end{array}$ & FVC, PEFR \\
\hline Pillastrini et al. ${ }^{26}$ & $\begin{array}{l}5 \\
C: 5 \\
4 / 1 \\
31.5 \pm 16.1\end{array}$ & $\begin{array}{l}4 \\
\text { C: } 4 \\
3 / 1 \\
52.2 \pm 17.6\end{array}$ & $\begin{array}{l}\text { C1-C7 } \\
\text { Mode NS }\end{array}$ & $\begin{array}{l}\text { C1-C7 } \\
\text { Mode NS }\end{array}$ & A & $A$ & $\begin{array}{l}\text { Acute } \\
\text { ward }\end{array}$ & FEV1, FVC, PEF \\
\hline Berney et al. ${ }^{39}$ & $\begin{array}{l}7 \\
C: 7 \\
7 / 0 \\
26.14\end{array}$ & $\begin{array}{l}7 \\
C: 7 \\
4 / 3 \\
29.43\end{array}$ & $\begin{array}{l}\text { C5-C6 } \\
\text { Mode C5 }\end{array}$ & $\begin{array}{l}\text { C5-C7 } \\
\text { Mode C5 } \\
\text { and C7 }\end{array}$ & A & A & $\begin{array}{l}\mathrm{SCl} \\
\text { specialist } \\
\mathrm{ICU}\end{array}$ & ICU LOS, MV duration, cost \\
\hline
\end{tabular}

Case series characteristics

\begin{tabular}{|c|c|c|c|c|c|}
\hline Citation & $\begin{array}{l}\text { Subjects } \\
N= \\
C / I \\
\text { Gender } M / F \\
\text { Mean age (years) }\end{array}$ & $\begin{array}{l}\text { Injury level } \\
\text { range mode }\end{array}$ & $A S I A$ & $\begin{array}{l}\text { Setting Site } \\
\text { Specialist } \\
\text { Centre }\end{array}$ & Outcome measure/description \\
\hline Romero et $a l^{38}$ & $\begin{array}{l}152 \\
119 / 33 \\
122 / 30 \\
40.86 \pm 1.86\end{array}$ & $\begin{array}{l}\text { C3-T12 } \\
\text { C3-5 }\end{array}$ & $A-D$ & $\begin{array}{l}\mathrm{SCI} \text { specialist } \\
\mathrm{ICU}\end{array}$ & $\begin{array}{l}\text { Pneumonia, duration of mechanical } \\
\text { ventilation and ICU length of stay } \\
\text { and mortality compared for tracheostomy } \\
\text { inserted before and after } 7 \text { days }\end{array}$ \\
\hline $\begin{array}{l}\text { Hornstein and } \\
\text { Ledsome }^{24}\end{array}$ & $\begin{array}{l}20 \\
16 / 4 \\
18 / 2 \\
25.5\end{array}$ & $\begin{array}{l}\text { C4-C7 } \\
\text { C5 }\end{array}$ & $\begin{array}{l}\text { A/incomplete } \\
\text { status NS }\end{array}$ & $\begin{array}{l}\mathrm{SCl} \text { specialist } \\
\text { acute ward }\end{array}$ & Pimax \\
\hline Berney et al. ${ }^{35}$ & $\begin{array}{l}71 \\
45 / 26 \\
46 / 25 \\
40.28 \pm 19.22\end{array}$ & $\begin{array}{l}\mathrm{C} 1-\mathrm{C} 8 \\
\mathrm{C} 5\end{array}$ & $A-D$ & $\begin{array}{l}\mathrm{SCl} \text { specialist } \\
\mathrm{ICU}\end{array}$ & $\begin{array}{l}\text { Tracheostomy timing after stabilization, } \\
\text { infection rate for anterior and posterior } \\
\text { approaches, and tracheostomy }\end{array}$ \\
\hline Stiller et al. ${ }^{27}$ & $\begin{array}{l}5 \\
\text { NS } \\
3 / 2 \\
34\end{array}$ & $\begin{array}{l}\text { C5-C7 } \\
\text { C5 }\end{array}$ & NS & $\begin{array}{l}\mathrm{SCl} \text { specialist } \\
\mathrm{ICU}\end{array}$ & TV, VC \\
\hline Lerman and Weiss ${ }^{25}$ & $\begin{array}{l}3 \\
2 / 1 \\
2 / 1 \\
31\end{array}$ & $\mathrm{C} 3 / 4$ & $A / B$ & $\begin{array}{l}\mathrm{SCI} \text { specialist } \\
\mathrm{ICU}\end{array}$ & VC, MIF, successful weaning \\
\hline Gardner et al. ${ }^{40}$ & $\begin{array}{l}44 \\
\text { NS } \\
37 / 7 \\
38.61\end{array}$ & $\begin{array}{l}\mathrm{C} 2-\mathrm{L} 1 \\
\mathrm{C} 4\end{array}$ & NS & $\begin{array}{l}\mathrm{SCl} \text { specialist } \\
\mathrm{ICU}\end{array}$ & Mortality, method of ventilation \\
\hline
\end{tabular}


Table 5 Continued

\begin{tabular}{|c|c|c|c|c|c|}
\hline Citation & $\begin{array}{l}\text { Subjects } \\
N= \\
C / I \\
\text { Gender } M / F \\
\text { Mean age } \\
\text { (years) }\end{array}$ & $\begin{array}{l}\text { Injury level } \\
\text { range mode }\end{array}$ & $A S I A$ & $\begin{array}{l}\text { Setting Site } \\
\text { Specialist } \\
\text { Centre }\end{array}$ & Outcome measure/description \\
\hline Hachen ${ }^{29}$ & $\begin{array}{l}188 \\
117 / 71 \\
\text { NS } \\
\text { NS }\end{array}$ & $\begin{array}{l}\text { C4-T1 } \\
\text { C6 } \\
\text { (Mode C: C7 } \\
\text { Mode I: C6) }\end{array}$ & $\begin{array}{l}\text { A/ } \\
\text { incomplete } \\
\text { status NS }\end{array}$ & $\begin{array}{l}\mathrm{SCl} \text { specialist } \\
\mathrm{ICU}\end{array}$ & $\begin{array}{l}\text { Mortality, airway management, } \\
\text { respiratory complication rate }\end{array}$ \\
\hline Harrop et al. ${ }^{36}$ & $\begin{array}{l}156 \\
156 / 0 \\
\text { NS } \\
38\end{array}$ & $\begin{array}{l}\mathrm{C} 2-\mathrm{C} 8 \\
\mathrm{C} 4\end{array}$ & $A$ & $\begin{array}{l}\mathrm{SCI} \text { specialist } \\
\mathrm{ICU}\end{array}$ & Airway management \\
\hline Velmahos et $a .^{32}$ & $\begin{array}{l}68 \\
41 / 27 \\
64 / 4 \\
34 \pm 16.5\end{array}$ & $\begin{array}{l}\text { NS } \\
32 / 68 \text { injuries } \\
\text { above } \mathrm{C5}\end{array}$ & $\begin{array}{l}\text { A/ } \\
\text { incomplete } \\
\text { status NS }\end{array}$ & $\begin{array}{l}\mathrm{SCl} \text { specialist } \\
\mathrm{ICU}\end{array}$ & $\begin{array}{l}\text { Airway management, risk factor } \\
\text { model to predict patients who } \\
\text { require intubation, incidence of } \\
\text { respiratory compromise }\end{array}$ \\
\hline Gupta et $a .^{23}$ & $\begin{array}{l}4 \\
4 / 0 \\
4 / 0 \\
36.25\end{array}$ & $\begin{array}{l}\mathrm{C} 5-\mathrm{C} 6 \\
\mathrm{C} 5\end{array}$ & A & $\begin{array}{l}\mathrm{SCl} \text { specialist } \\
\mathrm{ICU}\end{array}$ & Clinical use of a mini-tracheostomy \\
\hline Como et al. ${ }^{37}$ & $\begin{array}{l}119 \\
45 / 74 \\
93 / 26 \\
45\end{array}$ & $\begin{array}{l}\mathrm{C} 1-\mathrm{C} 8 \\
\text { NS }\end{array}$ & $\begin{array}{l}\text { A/ } \\
\text { incomplete } \\
\text { status NS }\end{array}$ & $\begin{array}{l}\text { SCI specialist } \\
\mathrm{ICU}\end{array}$ & $\begin{array}{l}\text { Airway management, mortality, } \\
\text { mechanical ventilation usage }\end{array}$ \\
\hline Green et $a .^{28}$ & $\begin{array}{l}162 \\
\text { NS } \\
143 / 19 \\
\text { NS }\end{array}$ & NS & NS & $\begin{array}{l}\mathrm{SCl} \text { specialist } \\
\mathrm{ICU}\end{array}$ & Mortality, respiratory complications \\
\hline Hassid et $a l^{30}$ & $\begin{array}{l}186 \\
108 / 78 \\
\text { NS } \\
35.92\end{array}$ & $\begin{array}{l}\text { C5-T1 mode } \\
\text { level not } \\
\text { able to be } \\
\text { determined }\end{array}$ & $A / B$ & $\begin{array}{l}\mathrm{SCl} \text { specialist } \\
\mathrm{ICU}\end{array}$ & $\begin{array}{l}\text { Airway management, respiratory } \\
\text { complications, ventilation hours, } \\
\text { ICU LOS, mortality }\end{array}$ \\
\hline Tromans et al. ${ }^{31}$ & $\begin{array}{l}28 \text { ( } 32 \text { treatment } \\
\text { occasions) } \\
24 / 8 \\
21 / 7 \\
40\end{array}$ & $\begin{array}{l}\mathrm{C} 2-\mathrm{T} 7 \\
\mathrm{C} 4\end{array}$ & $\mathrm{~A} / \mathrm{B} / \mathrm{C}$ & $\begin{array}{l}\text { SCI specialist } \\
\text { ICU }\end{array}$ & $\begin{array}{l}\text { Prophylactic: prevention of intubation } \\
\text { and mechanical ventilation } \\
\text { Weaning: weaning outcome }\end{array}$ \\
\hline
\end{tabular}

Abbreviations: AMS, ASIA motor score; ASIA, American spinal injury association classification; C, complete cervical spinal cord injury; CC, control cohort/cases; CG, control group; FEV1, forced expiratory volume in $1 \mathrm{~s}$; FVC, forced vital capacity; I, incomplete cervical spinal cord injury; IC, intervention cohort/cases; ICU, intensive care unit; IG, intervention group; LOS, length of stay; MV, mechanical ventilation; NS, not stated; PEF, peak expiratory flow; PEFR, peak expiratory flow rate; SCI, spinal cord injury. Mean age is at time of injury.

turning). The remaining three case series ${ }^{29,30,32}$ reported the incidence of respiratory complications associated with their approach to airway management. Overall, the incidence of pneumonia from these three reports was between 40 and 55\% with complete injuries having a higher incidence (60-70\%) than incomplete injuries (20-30\%).

\section{Airway management}

Seven studies reported outcomes concerning airway management. $^{29,30,32,34-37}$ Six studies were case series ${ }^{29,30,32,35-37}$ and one study was a cohort design. ${ }^{34}$ There were three issues addressed in the topic of airway management: (1) indications for intubation; (2) indications for tracheostomy; and (3) optimal timing of tracheostomy insertion after cervical stabilization surgery.

\section{Intubation}

One case series ${ }^{32}$ compared the characteristics of patients who were intubated with those who were not. They identified three independent risk factors for intubation: injury severity score, SCI above the level of C5, and a complete injury. Overall, $74 \%$ of the patients required intubation. Three other case series of varying quality reported similar rates of intubation ${ }^{29,30,37}$ although two of these studies included only patients with an injury of C5 and below. ${ }^{29,30}$ All studies reported a higher rate of intubation for complete injuries that varied from $74 \%{ }^{29,32}$ to above $90 \% .^{30,37}$

\section{Tracheostomy}

There were two factors that accounted for the incidence of tracheostomy: whether the injury was complete and the injury level being above or below C5. One case series ${ }^{36}$ reported the risk factors that predicted tracheostomy in patients with complete injures were age, pre-existing medical condition, pre-morbid lung disease, and injury level. The overall incidence of tracheostomy in these cases was $69 \%$. This was similar for other groups for complete injury ${ }^{30,34,37}$ 
Table 6 Results for case series

\begin{tabular}{|c|c|c|c|c|c|c|c|}
\hline \multirow[t]{2}{*}{ Citation } & \multirow[t]{2}{*}{ Treatment described } & \multicolumn{6}{|c|}{ Results } \\
\hline & & $\begin{array}{l}\text { Airway management } \\
\text { Overall } \\
\text { C } \\
I\end{array}$ & Respiratory complications & Physiological & LOS (days) & $\begin{array}{l}M V \text { incidencel } \\
\text { time (days) }\end{array}$ & Other \\
\hline $\begin{array}{l}\text { Romero } \\
\text { et } a .^{38}\end{array}$ & $\begin{array}{l}\text { Airway } \\
\text { management- } \\
\text { tracheostomy inserted } \\
\text { before or after day } 7\end{array}$ & & $\begin{array}{l}\text { Pneumonia: } \\
O=138 / 152(91 \%) \\
<7 \text { days } 62 / 71(87.3 \%) \\
>7 \text { days } 76 / 81(92.7 \%)\end{array}$ & & $\begin{array}{l}<7 \text { days } \\
36.52 \pm 1.6 \\
\text { days } \\
>7 \text { days } \\
54.58 \pm 3 \text { days } \\
P<0.001\end{array}$ & $\begin{array}{l}<7 \text { days } \\
26.07 \pm 1.69 \\
\text { days } \\
>7 \text { days } \\
48.75 \pm 3.45 \\
\text { days } P<0.005\end{array}$ & $\begin{array}{l}\text { Mortality: } \\
O=6 / 152 \\
(4 \%) \\
<7 \text { days } \\
1 / 71(1.4 \%) \\
>7 \text { days } \\
5 / 81(6 \%)\end{array}$ \\
\hline $\begin{array}{l}\text { Hassid } \\
\text { et al. }\end{array}$ & $\begin{array}{l}\text { Airway management of } \\
\text { patients between } \mathrm{C} 5 \\
\text { and } \mathrm{T} 1 \text { with no } \mathrm{TBI}\end{array}$ & $\begin{array}{l}\text { In }=127 / 186(68 \%) \\
T=88 / 186(47 \%) \\
C \\
\text { In }=97 / 108(90 \%) \\
\text { In to } T=73 / 97(75 \%) \\
T=73 / 108(68 \%) \\
I \\
\text { In }=30 / 78(38 \%) \\
\text { In to } T=15 / 30(50 \%) \\
T=15 / 78(19 \%)\end{array}$ & $\begin{array}{l}\text { Pneumonia: } O=92 / 186 \\
C \\
73 / 108(68 \%) \\
67 / 108 \text { In+6/11 non-In } \\
I \\
19 / 78(24 \%) \\
13 / 30 l+6 / 48 \text { non-I }\end{array}$ & & & & $\begin{array}{l}\text { Mortality: } \\
O=27 / 186 \\
C \\
26 / 108(24 \%) \\
16 / 97 \ln +10 / \\
11 \text { non- } \\
I \\
1 / 78(\ln )\end{array}$ \\
\hline $\begin{array}{l}\text { Velmahos } \\
\text { et } a l^{32}\end{array}$ & Airway management & $\begin{array}{l}\text { In }=50 / 68 \\
C=37 / 50(28 / 50 \\
\text { above } C 5) \\
\text { Risk factors for In } \\
\text { identified } \\
\text { ISS }>16 \\
(\mathrm{OR}=12.96,95 \% \mathrm{Cl} \text { : } \\
2.51,65.98, P=0.00) \\
\mathrm{SCl} \text { above } \mathrm{C} 5(\mathrm{OR} \\
8.71,95 \% \mathrm{Cl} 1.46- \\
51.87, P=0.02) \\
\mathrm{C}(\mathrm{OR} 7.96,95 \% \mathrm{Cl} \text { : } \\
1.61,37.13, P=0.01)\end{array}$ & $\begin{array}{l}\text { Pneumonia: } \\
27 / 68(40 \%) \\
\ln =25 / 50(50 \%)\end{array}$ & & & & $\begin{array}{l}\text { Mortality: } \\
O=11 / 68 \\
(16 \%) \\
\ln =11 / 50 \\
(22 \%)\end{array}$ \\
\hline $\begin{array}{l}\text { Como } \\
\text { et al. }\end{array}$ & Airway management & $\begin{array}{l}\text { In }=67 / 119(56 \%) \\
T=31 / 119(31 \%) \\
C \\
\text { In }=41 / 45(91 \%) \\
\text { In to } T=32 / 41(78 \%) \\
T=32 / 45(71 \%) \\
\text { All C C5 and above } \\
\text { (31) required } \\
\text { intubation and } \\
\text { tracheostomy. C6 and } \\
\text { below } \\
\text { In }=11 \\
T=6 \\
I \\
\text { In }=26 / 74(35 \%) \\
\text { In to } T=5 / 26(19 \%) \\
T=5 / 74(7 \%) \\
\text { Days (mean) to } T=10\end{array}$ & & & & $\begin{array}{l}\text { MVDC: } \\
O=20 / 119 \\
C \\
19 / 45 \\
17 / 19 \text { above } \\
C 5 \\
l \\
1 / 74\end{array}$ & \\
\hline $\begin{array}{l}\text { Harrop } \\
\text { et al. }\end{array}$ & Airway management & $\begin{array}{l}T=107 / 156(69 \%) \\
\text { Factors identified to } \\
\text { predispose to } T \\
\text { Age }(P=0.05) \\
\text { pre-existing medical } \\
\text { conditions }(P<0.05) \text {; } \\
\text { pre-morbid lung } \\
\text { disease }(P<0.05) ; \\
\text { injury level } \\
(P<0.0001) ; 88 / 107 \\
\text { between } C 4 \text { and } C 7\end{array}$ & & & & & \\
\hline Hachen $^{29}$ & Airway management & $\begin{array}{l}\text { In }=63 / 108(58 \%) \\
T=30 / 188(16 \%) \\
T \text { complications }=8 / 30 \\
(27 \%) \\
C\end{array}$ & $\begin{array}{l}\text { Respiratory insufficiency: } \\
O=101 / 188(54 \%) \\
C \\
82 / 117(70 \%) \\
I\end{array}$ & & & & \\
\hline
\end{tabular}


Table 6 Continued

\begin{tabular}{|c|c|c|c|c|c|c|c|}
\hline \multirow[t]{2}{*}{ Citation } & \multirow[t]{2}{*}{ Treatment described } & \multicolumn{6}{|c|}{ Results } \\
\hline & & $\begin{array}{l}\text { Airway management } \\
\text { Overall } \\
\text { C } \\
I\end{array}$ & Respiratory complications & Physiological & LOS (days) & $\begin{array}{l}M V \text { incidence/ } \\
\text { time (days) }\end{array}$ & Other \\
\hline & & $\begin{array}{l}\text { In }=50 / 117 \\
\text { In to } T 26 / 50(52 \%) \\
T=26 / 117(22 \%) \\
I \\
\text { In }=13 / 71(18 \%) \\
T=4 / 71(6 \%)\end{array}$ & 19/71 (27\%) & & & & \\
\hline $\begin{array}{l}\text { Berney } \\
\text { et al. }\end{array}$ & $\begin{array}{l}\text { Timing of } \\
\text { tracheostomy after } \\
\text { stabilization surgery } \\
\text { and comparison of } \\
\text { infection rates between } \\
\text { anterior and posterior } \\
\text { approach }\end{array}$ & $\begin{array}{l}T \text { timing (days) } \\
\text { anterior } 3.8 \pm 2.6 \\
\text { posterior } 3.1 \pm 2.7 \\
\text { anterior and posterior } \\
4.9 \pm 2.3 \text { days } \\
\text { No significant } \\
\text { difference between } \\
\text { timing }\end{array}$ & & & & & $\begin{array}{l}\text { Stabilization } \\
\text { approach: } \\
\text { Anterior }=32 \\
\text { Posterior }=15 \\
\text { Anterior and } \\
\text { posterior }=24 \\
\text { Incision } \\
\text { infection risk: } \\
\text { Posterior fusion } \\
\text { associated with } \\
\text { higher risk of } \\
\text { incision } \\
\text { infection (OR } \\
\text { 18.97, 95\% Cl } \\
2.31-155.54)\end{array}$ \\
\hline $\begin{array}{l}\text { Green } \\
\text { et al. }\end{array}$ & $\begin{array}{l}\text { Kinetic therapy } 2 \\
\text { weeks post acute injury }\end{array}$ & & $\begin{array}{l}\text { Pneumonitis/atelectasis: } \\
9 / 162(6 \%)\end{array}$ & & & & $\begin{array}{l}\text { Mortality: } \\
\text { 11/162 (7\%) }\end{array}$ \\
\hline $\begin{array}{l}\text { Tromans } \\
\text { et } \text { al. }^{31}\end{array}$ & $\begin{array}{l}\text { BIPAP for prevention of } \\
\text { ventilatory failure and } \\
\text { BIPAP to assist weaning } \\
\text { from full ventilation }\end{array}$ & & $\begin{array}{l}\text { Treatment of ventilatory } \\
\text { failure/avoiding intubation: } \\
\text { 10/17 (59\%) }\end{array}$ & & & $\begin{array}{l}\text { Successful } \\
\text { weaning: } \\
\text { From full MV } \\
13 / 15(87 \%)\end{array}$ & \\
\hline $\begin{array}{l}\text { Gupta } \\
\text { et al. }\end{array}$ & $\begin{array}{l}\text { Minitracheostomy use } \\
\text { to (1) treat retained } \\
\text { secretions and prevent } \\
\text { bronchcoscopy and } \\
\text { intubation (2) weaning } \\
\text { from tracheostomy }\end{array}$ & $\begin{array}{l}\text { Successful } \\
\text { decannulation }\end{array}$ & & $\begin{array}{l}\text { Clinical } \\
\text { improvement } \\
\text { in } \mathrm{PaO}_{2} / \mathrm{FiO}_{2} \\
\mathrm{MD} 95 \% \\
\mathrm{Cl}=103 \\
(-97.34 \\
303.34)\end{array}$ & & & $\begin{array}{l}\text { CXR } \\
\text { CXR clearing }\end{array}$ \\
\hline $\begin{array}{l}\text { Lerman } \\
\text { and } \\
\text { Weiss } \\
25\end{array}$ & $\begin{array}{l}\text { Respiratory protocol: } \\
\text { respiratory muscle } \\
\text { training, IPPB, chest } \\
\text { percussion and } \\
\text { vibration, postural } \\
\text { drainage, assisted } \\
\text { cough, suctioning }\end{array}$ & $\begin{array}{l}\text { All patients were } \\
\text { successfully } \\
\text { weaned }\end{array}$ & & $\begin{array}{l}\text { Increase VC } \\
\mathrm{MD} 95 \% \\
\mathrm{Cl}=410 \\
(362.90 \\
1182.90) \mathrm{ml} \\
\text { and increase } \\
\mathrm{MIF}, \mathrm{MD} 95 \% \\
\mathrm{Cl}=-10.33 \\
(-32.68 \\
12.02)\end{array}$ & & & \\
\hline $\begin{array}{l}\text { Stiller } \\
\text { et al. }{ }^{27}\end{array}$ & $\begin{array}{l}\text { IPPB } 2 \text { hourly four reps } \\
\text { of six breaths with } \\
30-60 \text { s of relaxed } \\
\text { breathing }\end{array}$ & & & $\begin{array}{l}\text { IPPB volume } \\
\text { increased } \\
(P<0.001) \\
\text { Post IPPB Vt } \\
\text { not different } \\
\text { VC increased } \\
43 \mathrm{ml} \\
(P<0.02)\end{array}$ & & & \\
\hline $\begin{array}{l}\text { Gardner } \\
\text { et al. }\end{array}$ & $\begin{array}{l}\text { Provision of } \\
\text { mechanical ventilation } \\
\text { for high quadriplegia }\end{array}$ & & & & & & $\begin{array}{l}\text { Mortality: } \\
14 / 44 \text { (32\%) } \\
\text { died on first } \\
\text { admission }\end{array}$ \\
\hline $\begin{array}{l}\text { Hornstein } \\
\text { and } \\
\text { Ledsome } \\
24\end{array}$ & $\begin{array}{l}\text { Ventilatory } \\
\text { muscle } \\
\text { training protocol }\end{array}$ & & & $\begin{array}{l}\text { Increase Pimax } \\
\text { MD } \\
95 \% \\
\mathrm{Cl}=-13.55 \\
(-28.87,1.77)\end{array}$ & & & \\
\hline
\end{tabular}


Table 6 Continued

Results for case control or cohort study

\begin{tabular}{|c|c|c|c|c|c|c|c|c|}
\hline \multirow[t]{2}{*}{ Citation } & \multirow[t]{2}{*}{ Intervention } & \multirow[t]{2}{*}{ Control/cohort } & \multicolumn{6}{|c|}{ Results } \\
\hline & & & $\begin{array}{l}\text { Airway } \\
\text { management } \\
\text { Overall } \\
\mathrm{Cl}\end{array}$ & $\begin{array}{l}\text { Respiratory } \\
\text { complications }\end{array}$ & Physiological & $\begin{array}{l}\operatorname{LOS} \\
\text { (days) }\end{array}$ & $\begin{array}{l}\text { MV } \\
\text { (incidence/time) }\end{array}$ & Other \\
\hline $\begin{array}{l}\text { Gregoretti } \\
\text { et al. }\end{array}$ & $\begin{array}{l}\text { Transtracheal } \\
\text { open ventilation }\end{array}$ & $\begin{array}{l}\text { Conventional } \\
\text { mechanical } \\
\text { ventilation }\end{array}$ & & & $\begin{array}{l}\mathrm{PaO}_{2} / \mathrm{FiO}_{2}, \mathrm{PaCO}_{2}, \mathrm{RR} \\
\text { not different after } 1 \\
\text { and } 24 \mathrm{~h} \text {, pressure time } \\
\text { product }(\mathrm{PTP}) \text { of } \\
\text { oesophageal pressure } \\
\text { less after } 24 \mathrm{~h} P<0.05 \\
\mathrm{MD} 95 \% \mathrm{Cl} P / \mathrm{F}=10 \\
(-47.31,67.31) \\
\mathrm{PaCO}_{2}=-2.2 \\
(-7.18,2.78) \\
R R=-3 \\
(-6.82,0.82) \\
\mathrm{PTP}=-63 \\
(-29.39,-96.61)\end{array}$ & & & \\
\hline $\begin{array}{l}\text { Braun } \\
\text { et al. }\end{array}$ & $\begin{array}{l}\text { Assisted FVC } \\
\text { and cough }\end{array}$ & $\begin{array}{l}\text { Unassisted } \\
\text { FVC and cough }\end{array}$ & & & $\begin{array}{l}\text { Peak flow rate } \\
\text { increased } 13.8 \% \text { with } \\
\text { assistance } P<0.01 \text {, } \\
\text { no significant } \\
\text { improvement in } \\
\text { volume }\end{array}$ & & & \\
\hline $\begin{array}{l}\text { McMichan } \\
\text { et al. }\end{array}$ & $\begin{array}{l}\text { Intensive } \\
\text { respiratory care } \\
\text { protocol: } \\
\text { positioning, } \\
\text { deep breathing, } \\
\text { incentive } \\
\text { spirometry, } \\
\text { chest percussion, } \\
\text { assisted cough }\end{array}$ & $\begin{array}{l}\text { Historical } \\
\text { standard } \\
\text { care }\end{array}$ & $\begin{array}{l}\text { Decrease } \\
\text { tracheostomy } \\
\text { use } 2 / 22 \text { and } \\
6 / 22 \text { for } \\
\text { control } \\
\mathrm{ARR}=0.18, \\
95 \% \mathrm{Cl} \\
(-0.05,0.4) \\
\mathrm{RRR}=0.67, \\
95 \% \mathrm{Cl} \\
(-0.19,1.47) \\
\text { NNT }=6\end{array}$ & $\begin{array}{l}\text { Atelectasis: } \\
\text { reduction in } \\
\text { atelectasis } 4 / 22 \\
\text { intervention } \\
\text { and } 12 / 22 \text { in } \\
\text { control. } \\
\text { ARR }=0.36 \text {, } \\
95 \% \mathrm{Cl} \\
(0.08,0.58) \\
\mathrm{RRR}=0.67 \text {, } \\
95 \% \mathrm{Cl} \\
(0.15,1.06) \\
\text { NNT }=3\end{array}$ & & & $\begin{array}{l}\text { Decrease need } \\
\text { for } \mathrm{MV} 3 / 22 \text { and } \\
9 / 22 \text { in controls. } \\
\mathrm{ARR}=0.27,95 \% \\
\mathrm{Cl}(0.01,0.49) \\
\mathrm{RRR}=0.67,95 \% \\
\mathrm{Cl}(0.02,1.21) \\
\mathrm{NNT}=4\end{array}$ & $\begin{array}{l}\text { Morality: } \\
\text { Reduction on } \\
\text { mortality } 0 / 22 \\
\text { mortality in } \\
\text { intervention } 9 / 22 \\
\text { in control } \\
\text { ARR }=0.4,95 \% \mathrm{Cl} \\
(0.18,0.61) \\
\text { RRR }=1,95 \% \mathrm{Cl} \\
(0.44,1.50) \\
\text { NNT }=2\end{array}$ \\
\hline $\begin{array}{l}\text { Vitaz } \\
\text { et } \text { al. }^{33}\end{array}$ & $\begin{array}{l}\text { Clinical } \\
\text { pathway -4 } \\
\text { hourly chest } \\
\text { physiotherapy; } \\
\text { fixation within } \\
2 \text { days with } \\
\text { tracheostomy if } \\
\text { required at day } 4\end{array}$ & $\begin{array}{l}\text { Historical } \\
\text { standard care }\end{array}$ & & $\begin{array}{l}\text { Decreased } \\
\text { episodes of } \\
\text { pneumonia/ } \\
\text { patient } \\
P=0.05\end{array}$ & & $\begin{array}{l}\text { ICU LOS: } \\
\text { Decreased } \\
\text { mean } \\
\text { difference } 95 \% \\
\mathrm{Cl} \text { (days) } 6.8 \\
\text { (-0.17, } 13.77 \\
\text { Hospital LOS: } \\
\text { Mean } \\
\text { difference } 95 \% \\
\mathrm{Cl} \text { (days) } \\
\text { decreased } 11.5 \\
(3.49,19.51)\end{array}$ & $\begin{array}{l}\text { MV days } \\
\text { decrease mean } \\
\text { difference } 95 \% \\
\mathrm{Cl} \text { (days) } \\
6(-0.56,12.56)\end{array}$ & $\begin{array}{l}\text { Costs: } \\
\$ 20000 \text { per } \\
\text { patient saved }\end{array}$ \\
\hline $\begin{array}{l}\text { Berney } \\
\text { et al. }\end{array}$ & $\begin{array}{l}\text { Intensive } \\
\text { physiotherapy } \\
\text { to prevent } \\
\text { tracheostomy }\end{array}$ & $\begin{array}{l}\text { Patients who } \\
\text { received } \\
\text { tracheostomy }\end{array}$ & & & & $\begin{array}{l}\text { ICU LOS: } \\
\text { Mean } \\
\text { difference } 95 \% \\
\mathrm{CI} \text { decrease } \\
8.71(1.75, \\
15.67)\end{array}$ & $\begin{array}{l}\text { MV days } \\
\text { Decrease } \\
P=0.006 \text { Mean } \\
\text { difference } 95 \% \\
\mathrm{Cl} \text { decrease } \\
8 \text { days } \\
(4.66,11.34)\end{array}$ & $\begin{array}{l}\text { Cost: } \\
\text { Saving of } \\
\$ 1270 \text { per patient } \\
\text { per day }\end{array}$ \\
\hline $\begin{array}{l}\text { Bellamy } \\
\text { et al. }{ }^{34}\end{array}$ & $\begin{array}{l}\text { Airway } \\
\text { management }\end{array}$ & $\begin{array}{l}\text { Cervical spine } \\
\text { fracture } \\
\text { with no } \\
\text { neurology }\end{array}$ & $\begin{array}{l}\mathrm{OT}=31 / 54 \\
(57 \%) \\
\mathrm{CCSCl} 23 / 30 \\
(77 \%) \\
\mathrm{ICSCl}=8 / 24 \\
(33 \%) \\
\text { T performed } \\
\text { within } 3 \text { days } \\
\text { No neurology } \\
\text { group } \\
0 \mathrm{~T}=0\end{array}$ & & & & & $\begin{array}{l}\text { Mortality: } \\
O=15 / 54(28 \%) \\
\text { CCSCI12/30 (40\%) } \\
\text { ICSCI 5/24 (21\%) }\end{array}$ \\
\hline
\end{tabular}


Table 6 Continued

Results from randomized controlled trials/randomized trials

\begin{tabular}{|c|c|c|c|c|c|}
\hline Author date & $n$ & Setting & Intervention & Control/comparison & Result \\
\hline Pillastrini et al. ${ }^{26}$ & 9 & Acute & $\begin{array}{l}\text { Manual respiratory kinesitherapy+ } \\
\text { mechanical insufflations/exsufflation } \\
\text { inhale pressure } 15 \mathrm{cmH}_{2} \mathrm{O} \text {, exhale } \\
\text { pressure } 45 \mathrm{cmH}_{2} \mathrm{O}\end{array}$ & $\begin{array}{l}\text { Manual respiratory kinesitherapy for } \\
10 \text { treatments: postural drainage, } \\
\text { PEP assisted cough, AMBU bag, } \\
\text { endoscopic bronchoaspiration }\end{array}$ & $\begin{array}{l}\text { Increase FVC, FEV1 }(P=0.0001) \text {, } \\
\text { increased PEF }(P=0.0093) \\
\text { MD and } 95 \% \mathrm{Cl} \text { in FVC } 0.09 \text { I } \\
(-0.23,0.41) \\
\text { MD and } 95 \% \mathrm{Cl} \text { for FEV1 } 0.07 \text { I } \\
(-0.14,0.28)\end{array}$ \\
\hline
\end{tabular}

Abbreviations: ARR, absolute risk reduction; BiPAP, bi-level airway pressure; $\mathrm{C}$, complete spinal cord injury; $\mathrm{CCSCl}$, complete cervical spinal cord injury; 95\% Cl, 95\% confidence interval; CXR, chest radiograph; FEV1, forced expiratory volume in $1 \mathrm{~s}$; FVC, forced vital capacity; I, incomplete spinal cord injury; ICSCI, incomplete cervical spinal cord injury; ICU, intensive care unit; In, intubated; IPPB, intermittent positive pressure breathing; ISS, injury severity score; LOS, length of stay; MD, mean difference; MIF, maximal inspiratory force; MV, mechanical ventilation; MVDC, mechanical ventilation at discharge; NNT, number needed to treat; PEF, peak expiratory flow; PEP, positive expiratory pressure; RR, respiratory rate; RRR, relative risk reduction; SCl, spinal cord injury; T, tracheostomy; TBI, traumatic brain injury; VC, vital capacity; Vt, tidal volume.

except for one low quality ${ }^{29}$ study that reported a much lower incidence of tracheostomy insertion (22\%). The incidence of tracheostomy insertion in incomplete injuries was low (6-33\%). ${ }^{29,30,34,37}$ For complete injuries above the C5 level the incidence of tracheostomy was between $81^{36}$ and $83 \%{ }^{37}$ compared with $49^{36}-60 \%{ }^{37}$ for complete injuries C5 and below.

\section{Tracheostomy timing}

Three studies reported on the timing of tracheostomy insertion after injury, ${ }^{34,37,38}$ which varied from 3 days ${ }^{34}$ to 10 days $^{37}$ and one study ${ }^{35}$ reported timing post cervical spine anterior surgical stabilization. This case series of 78 patients $^{35}$ also examined the relationship between timing of tracheostomy insertion and the risk of cross infection at the anterior stabilization incision site, which is an important clinical consideration. These authors reported that tracheostomy insertion as early as day 4 post anterior cervical stabilization did not appear to pose a risk of cross infection. Another case series ${ }^{38}$ showed that tracheostomy inserted before day 7 reduced the duration of mechanical ventilation and ICU length of stay.

\section{ICU and hospital length of stay and costs}

Two good quality studies and one case series ${ }^{38}$ used ICU and or hospital length of stay as an outcome. ${ }^{33,39}$ Berney et al. using a case-control design showed in comparable groups of selected patients that intensive physiotherapy can prevent the need for tracheostomy and reduce length of stay in ICU (mean difference $=8.71$ days, 95\% CI 3.49, 19.51) therefore reducing costs. Romero et al. reported that tracheostomy insertion before day 7 reduced both duration of mechanical ventilation (mean difference $=22.68$ days, 95\% CI 21.79, 23.57) and ICU length of stay (mean difference18.06 days, $95 \%$ CI 17.29, 18.83). Vitaz et al. reported a reduction in ICU length of stay (mean difference $=6.8$ days, 95\% CI -01.7 , 13.77) and hospital length of stay (mean difference $=11.5$ days, 95\% CI 3.49, 19.51) saving a mean of $\$ 20000$ USD per patient using a clinical pathway to direct all aspects of acute care.

\section{Mechanical ventilation and weaning}

The incidence or the duration of mechanical ventilation was described in six studies. There was moderate evidence that intensive respiratory protocols alter the need for mechanical ventilation. McMichan et al. showed a reduced need for mechanical ventilation $(\mathrm{ARR}=0.27,95 \% \mathrm{CI} 0.01,0.49)$ with a number needed to treat of four and an author of this review (SB), showed a reduction in the duration of mechanical ventilation (mean difference $=8$ days $95 \%$ CI $4.66,11.34$ ). ${ }^{39}$ Vitaz et al. similarly showed that a protocolized care pathway compared with historical standard care can reduce the duration of mechanical ventilation (mean difference 6 days $95 \%$ CI $-0.56,12.56)$. Overall, there was limited evidence to support the use of NIV to reduce the duration of mechanical ventilation. $^{31}$ One case series ${ }^{38}$ reported that tracheostomy timing either before or after day 7 was independently associated with duration of mechanical ventilation.

\section{Mortality}

Six studies reported mortality rates associated with treatment. ${ }^{10,28,30,32,34,40}$ A respiratory care protocol ${ }^{10}$ showed a reduction in mortality compared with a historical control group who appeared to receive no specific respiratory treatment $(\mathrm{ARR}=0.4,95 \% \mathrm{CI} 0.18,0.61)$ with a number needed to treat of two. There is no high quality evidence to support the use of kinetic therapy to reduce mortality. Three studies ${ }^{30,32,34}$ reported mortality rates between $16^{32}$ and $28 \% .^{34}$

\section{Discussion}

There were three main outcomes of this review. First, respiratory complications are prevalent, however treatment protocols that include a combination of techniques, applied frequently, appear to be the most effective in preventing respiratory complications, reducing mortality, ICU length of 
stay, and the duration of mechanical ventilation and improving the physiological status of the patient. ${ }^{10,25,39}$ These treatment techniques include positioning, assist coughing/suctioning, and lung volume restoration therapy. Second, managing the processes of care with a clinical pathway is cost effective and appear also to be effective in reducing the incidence and severity of respiratory complications, the duration of mechanical ventilation, and ICU length of stay. ${ }^{33}$ Third, intubation, mechanical ventilation, and tracheostomy remain the mainstay of respiratory management especially for injures American spinal injury association classification (ASIA) A injures above the C5 level.

Most studies in this review were either case series or cohorts studies with historical controls and all were from single centres. The results are therefore prone to bias and the only RCT rated poorly with little consideration of statistical power, blinding, or comparability between groups. In many studies there was inadequate classification of incomplete SCI, which may reflect the difficulties associated with neurological assessment in the early phase of injury because of instability of baseline neurological examination. ${ }^{41}$ This in part can be influenced by the effects of cord swelling or bleeding that can result in patients losing a neurological level within the first few days. ${ }^{37}$ It has been suggested if possible that patients not be recruited to trials until $48 \mathrm{~h}$ post injury so a more reliable base line neurological level can be established. ${ }^{41}$ However, management for the prevention of respiratory complications must begin immediately ${ }^{2}$ making research in this phase of SCI more complex.

This review has found that evidence addressing the acute respiratory management of SCI comprises predominantly low quality trials. In part, this is an illustration of the difficulties associated with trial design in the early phases of SCI. Performing the gold standard RCT in this population is problematic because of heterogeneity of injury, the lack of a consistent approach to management, ${ }^{42}$ relatively small patient numbers, ${ }^{43}$ and instability of baseline neurological assessment. ${ }^{41}$ To overcome some of these issues, several studies in this review included only complete injuries. However, efficacy of interventions in this subgroup may not be able to be generalized to incomplete injuries. Largescale multi-centre RCTs are possible in $\mathrm{SCI}^{4}{ }^{44}$ however, they are major organizational undertakings that require stringent inclusion criteria to account for other trauma and comorbidity and on-going close monitoring of sites and treatment protocols during the trial. ${ }^{41}$ Alternative designs and strategies such as cluster randomization and adaptive randomization may enable phase III controlled trials to be performed. ${ }^{41}$ Despite the difficulties that injury heterogeneity, generalizability, and design pose, respiratory complications are predictable both in their nature and time course. Future research could investigate the efficacy of specific interventions and protocols that address the known risk factors and time course such as specific protocols for upper cervical lesions for the prevention of pneumonia.

Transtracheal open ventilation is a novel treatment approach to both mechanical ventilation and airway management and conveys the potential benefits of communication and oral intake to the patients. It warrants further longer-term comparison with conventional mechanical ventilation and tracheostomy measuring outcomes such as respiratory complications, wound infection, duration of mechanical ventilation, and ICU length of stay.

Airway management was a strong theme of the reports included in this review. There was little conjecture about the airway management of complete cervical injuries above the level of $\mathrm{C} 4$ and most reports concentrated on lower complete and incomplete injuries. Future multi-centre studies examining the factors that predict the need for tracheostomy in lower cervical or incomplete injuries that consider the role of NIV are required. In the future a guideline for airway management in this population that included recommendations for timing of tracheostomy insertion would be of value particularly for patients who are managed outside a specialized spinal unit.

The role of NIV in the ICU continues to grow as evidence accumulates regarding the prevention of post-extubation respiratory failure and the successful treatment of respiratory failure. ${ }^{45}$ The role of NIV in the respiratory management of acute SCI at the level of $\mathrm{C} 4$ and below warrants further investigation. These roles of NIV in the treatment of respiratory failure and weaning would appear plausible given the indications for NIV in other adult ICU populations. ${ }^{45,46}$ However, the use of NIV as a substitute for conventional mechanical ventilation would be unlikely to receive widespread support in the ICU community and at best would be case dependent. Given the results of this review, future research using NIV should be examined in combination with other therapies such as an intensive respiratory care protocol and outcomes should include ICU and hospital length of stay and the use and duration of intubation, tracheostomy, and mechanical ventilation.

This review had several limitations. By restricting management to the first 6 weeks after injury, papers may have been omitted that discussed pertinent issues to the acute phase such as weaning and the role of early respiratory muscle training. However, the focus of this review was the acute period of reversible respiratory failure that occurs as a result of the SCI and the ensuing spinal shock. Prolonged weaning is usually associated with high cervical injuries and is not a respiratory complication but a consequence of injury severity. Long-term weaning strategies alone could be a subject of a future systematic review. Several papers were included that were greater than 20-years-old, which limited their generalizability to current practice. This was particularly evident in results pertaining to overall ${ }^{28,34}$ and hospital mortality $^{10}$ where advances in medical practice in early resuscitation, ICU, and post-ICU care have resulted in improved rates of survival. ${ }^{47}$ It was decided to include these papers as these data are referred to in recent narrative reviews and form the basis of current treatment recommendations. ${ }^{48,49}$

In conclusion, this systematic review has revealed that a clinical pathway with a structured respiratory protocol that includes a combination of treatment techniques provided regularly is effective in reducing respiratory complications and the cost of acute care. Future consideration of collaboration and trial design with particular emphasis on inclusion 
criteria especially neurological severity and injury is necessary so trials of sufficient quality and power are performed that address effective ways of improving overall pulmonary function and performance and the prevention of respiratory complications.

\section{Conflict of interest}

The authors declare no conflict of interest.

\section{Acknowledgements}

We thank Ms Anne McLean for her assistance and also the Victorian Neurotrauma Initiative for the fellowship for Susan Berney.

\section{References}

1 Royster RA, Barboi C, Peruzzi WT. Critical care in the acute cervical spinal cord injury. Top Spinal Cord Inj Rehabil 2004; 9: 11-32.

2 Claxton R, Wong D, Chung F, Fehlings M. Predicotrs of hospital mortality and mechanical ventilation in patients with cevical spinal cord injury. Can J Anaesth 1998; 45: 114-149.

3 Jackson A, Groomes T. Incidence of respiratory complications following spinal cord injury. Arch Phys Med Rehabil 1994; 75: 270-275.

4 Winslow C, Bode R, Felton D, Chen D, Meyer P. Impact of respiratory complications on length of stay and hospital costs in acute cervical spine injury. Chest 2002; 121: 1548-1554.

5 Ball P. Critical care of spinal cord injury. Spine 2001; 26: S27-S30.

6 Mansel J, Norman J. Respiratory complications and management of spinal cord injuries. Chest 1990; 97: 1446-1452.

7 Ditunno JF, Little JW, Tessler A, Burns AS. Spinal shock revisited: a four-phase model. Spinal Cord 2004; 42: 383-395.

8 Lemmons $\mathrm{V}$, Wagner $\mathrm{F}$. Respiratory complications after spinal cord injury. Spine 1994; 19: 2315-2320.

9 Wuermser L, Ho C, Chiodo AE, Priebe MM, Kirshblum SC, Scelza $\mathrm{WH}$. Acute care management of traumatic and nontraumatic injury. Arch Phys Med Rehabil 2007; 88: S56-S61.

10 McMichan J, Michel L, Westbrook P. Pulmonary dysfunction following traumatic tetraplegia. J Am Med Assoc 1980; 243: 528-531.

11 Wallbom AS, Naran B, Thomas E. Acute ventilator management and weaning in individuals with high tetraplegia. Top Spinal Cord Inj Rehabil 2005; 10: 1-7.

12 Berlly M, Shem K. Respiratory management during the first five days after spinal cord injury. J Spinal Cord Med 2007; 30: 309-318.

13 Consortium for Spinal Cord Medicine. Respiratory management following spinal cord injury: a clinical practice guideline for health care professionals. 2005 [cited 10/11/2008]; available from http://www.guideline.gov/summary/pdf.aspx?doc_id=7198\&stat= $1 \&$ string=spinal+AND+cord+AND+injury.

14 National Health Medical Research Council N. NHMRC additional levels of evidence and grades for recommendations for developers of guidelines. Stage 2 consultation. 2008 [cited 3/12/2008]; available from http://www.nhmrc.gov.au/guidelines/consultations.

15 Maher CG, Sherrington C, Herbert RD, Moseley AM, Elkins M. Reliability of the PEDro Scale for rating quality of randomized controlled trials. Phys Ther 2003; 83: 713-721.

16 Wells GA, O'Connell D, Peterson J, Welch V, Tugwell P. The Newcastle-Ottowa Scale (NOS)for assessing the quality of nonrandomised studies in meta-analyses. 2000 [cited 3/12/2008]; available from http://www.ohri.ca.programs/clinicalepidemiology/ oxford/htm

17 Stevens RD, Dowdy DW, Micheals RK, Mendez-Tellez PA, Pronovost PJ, Needham DM. Neuromuscular dysfunction ac- quired in critical illness: a systematic review. Intensive Care Med 2007; 33: 1876-1891.

18 van Tulder M, Furlan A, Bombardier C, Bouter L. Updated method guidelines fir systematic reviews in the cochrane collaboration back review group. Spine 2003; 28: 1290-1299.

19 Prins MR, van der Wurff P. Females with patellofemoral pain have weak hip muscles: a systematic review. Aust J Physiother 2009; 55: 9-15.

20 Van Houtte S, Vanlandewijck Y, Gosselink R. Respiratory muscle training in persons with spinal cord injury: a systematic review. Res Med 2006; 100: 1886-1895.

21 Braun S, Giovannoni R, O'Connor M. Improving the cough in patients with spinal cord injury. Am J Phys Med 1984; 63: 1-10.

22 Gregoretti C, Olivieri C, Navalesi P. Physiologic comparison between conventional mechanical ventilation and transtracheal open ventilation in acute traumatic quadriplegic patients. Crit Care Med 2005; 33: 1114-1118.

23 Gupta A, McClelland R, Evans A, El Masri WS. Minitracheotomy in the early respiratory management of patients with spinal injuries. Paraplegia 1989; 27: 269-277.

24 Hornstein S, Ledsome JR. Ventilatory muscle training in acute tetraplegia. Physiother Can 1986; 38: 145-149.

25 Lerman RM, Weiss MS. Progressive resistance exercsie in weaning high quadriplegics from the ventilator. Paraplegia 1987; 25: 130-135.

26 Pillastrini P, Bordini S, Bazzochi G, Belloni G, Menarini M. Study of the effectiveness of bronchial clearance in subjects with spinal cord injuries, examination of a rehabilitation programme involving mechanical insufflations and exsufflation. Spinal Cord 2006; 44: 614-616.

27 Stiller K, Simionato R, Rice K, Hall B. The effect of intermittent positive pressure breathing on lung volumes in acute quadriparesis. Paraplegia 1992; 30: 121-126.

28 Green BA, Greek KL, Klose KJ. Kinetic therapy for spinal cord injury. Spine 1983; 8: 722-728.

29 Hachen HJ. Idealized care of the acutely injured spinal cord in Switzerland. J Trauma 1977; 17: 931-936.

30 Hassid VJ, Schinco MA, Tepas JJ, Griffen MM, Murphy TL, Fryberg ER et al. Definitive establishment of airway control is critical for optimal outcome in lower cervical spinal cord injury. J Trauma 2008; 65: 1328-1332.

31 Tromans AM, Mecci M, Barrett FH, Ward TA, Grundy DJ. The use of the BiPAP biphasic positive airway pressure system in acute spinal cord injury. Spinal Cord 1998; 36: 481-484.

32 Velmahos G, Toutouzas K, Chan L, Tillou A, Rhee P, Murray J et al. Intubation after cervical spinal cord injury: to be done selectively or routinely? Am Surg 2003; 69: 891-894.

33 Vitaz TW, Mcllvoy L, Raque GH, Spain DA, Shields CB. Development and implementation of a clinical pathway for spinal cord injuries. J Spinal Disord 2001; 14: 271-276.

34 Bellamy R, Pitts FW, Stauffer ES. Respiratory complications in traumatic tetraplegia. Analysis of 20 years' experience. J Neurosurg 1973; 39: 596-600.

35 Berney S, Opdam H, Bellomo R, Liew S, Skinner E, Egi M et al. An assessment of early tracheostomy after anterior cervical stabilization in patients with acute cervical spine trauma. J Trauma 2008; 64: 749-753.

36 Harrop J, Sharan A, Scheid E, Vaccaro A, Przybylski G. Tracheostomy placement in patients with complete cervical spinal cord injuries: American spinal injury association grade A. J Neurosurg 2004; 100: 20-23.

37 Como JJ, Sutton ERH, McCunn M, Dutton RP, Johnson SB, Aarabi $\mathrm{B}$ et al. Characterizing the need for mechanical ventilation following cervical spinal cord injury with neurologic deficit. J Trauma 2005; 59: 912-916.

38 Romero J, Var A, Gambarrutta C, Oliviero A. Tracheostomy timing in traumatic spinal cord injury. Eur Spine J 2009; 18: 1542-1547.

39 Berney S, Stockton K, Denehy L, Berlowitz D. Can early extubation and intensive physiotherapy decrease length of stay of acute quadriplegic patients in intensive care? A retrospective case control study. Physiother Res Int 2002; 7: 14-22.

40 Gardner BP, Watt JWH, Krishna KR. The artificial ventilation of acute spinal cord damaged patients: a retrospective study of fortyfour patients. Paraplegia 1986; 24: 208-220. 
41 Tator $\mathrm{CH}$. Review of treatment trials in human spinal cord injury: issues, difficulties and recommendations. Neurosurgery 2006; 59: 957-987.

42 Hadley MN. Management of acute spinal cord injuries in an intensive care unit or other monitored setting. Neurosurgery 2002; 50: S7-S51.

43 Sheel AW, Reid WD, Townson AF, Ayas N. Respiratory management following spinal cord injury. [Internet] 2006, 2005, cited 6/11/2008; available from http://www.icord/scire.

44 Bracken MB, Shepard MJ, Holford TR, Leo-Summers L, Aldrich EF, Fazl M et al. Administration of methylprednisolone for 24 or $48 \mathrm{~h}$ or tirilazad mesylate for $48 \mathrm{~h}$ in the treatment of acute spinal cord injury. Results of the third national acute spinal cord injury randomized controlled trial. national acute spinal cord injury study. J Am Med Assoc 1997; 277: 1597-1604.
45 Peñuelas O, Frutos-Vivar F, Esteban A. Noninvasive positivepressure ventilation in acute respiratory failure. Can Med Assoc J 2007; 177: 1211-1218.

46 Burns KEA, Adhikari NKG, Keenan SP, Meade M. Use of noninvasive ventilation to wean critically ill adults off invasive ventilation: meta-analysis and systematic review. $\mathrm{Br}$ Med J 2009; 338: b1574.

47 DeVivo MJ, Krause S, Lammertse DP. Recent trends in mortlaity and causes of death among persons with spinal cord injury. Arch Phys Med Rehabil 1999; 80: 1411-1419.

48 Urdaneta F, Layon J. Respiratory complications in patients with traumatic cervical spine injuries: case report and review of the literature. J Clin Anesth 2003; 15: 398-405.

49 Winslow C, Rozovsky J. Effect of spinal cord injury on the respiratory system. Am J Phys Med Rehabil 2003; 82: 803-814. 